\title{
One Week of Motor Adaptation Induces Structural Changes in Primary Motor Cortex That Predict Long-Term Memory One Year Later
}

\author{
Sofia M. Landi, Federico Baguear, and Valeria Della-Maggiore \\ Department of Physiology and Biophysics, School of Medicine, University of Buenos Aires, C1121ABG Buenos Aires, Argentina
}

The neural bases of motor adaptation have been extensively explored in human and nonhuman primates. A network including the cerebellum, primary motor cortex, and posterior parietal cortex appears to be crucial for this type of learning. Yet, to date, it is unclear whether these regions contribute directly or indirectly to the formation of motor memories. Here we trained subjects on a complex visuomotor rotation associated with long-term memory (in the order of months) to identify potential sites of structural plasticity induced by adaptation. One week of training led to (1) an increment in local gray matter concentration over the hand area of the contralateral primary motor cortex and (2) an increase in fractional anisotropy in an area underneath this region that correlated with the speed of learning. Moreover, the change in gray matter concentration measured immediately after training predicted improvements in the speed of learning during readaptation 1 year later. Our study suggests that motor adaptation induces structural plasticity in primary motor circuits. In addition, it provides the first piece of evidence indicating that early structural changes induced by motor learning may impact on behavior up to 1 year after training.

\section{Introduction}

Motor adaptation is a type of motor learning that allows maintaining accurate movements in the presence of environmental or internal perturbations by adjusting motor output. The neural substrates of motor adaptation have been extensively explored in human and nonhuman primates. Cerebellar damage significantly impairs this type of learning early during adaptation, and also hinders retention (Martin et al., 1996; Smith and Shadmehr, 2005; Criscimagna-Hemminger et al., 2010). The posterior parietal cortex (PPC) and the primary motor cortex (M1) appear to be recruited during late stages of adaptation (Della-Maggiore et al., 2004; Orban de Xivry et al., 2011), whereas M1 may be relevant for memory retention (Li et al., 2001; Paz et al., 2003) and for relearning $24 \mathrm{~h}$ later (Richardson et al., 2006). Determining whether these regions undergo neural plasticity or contribute to the formation of motor memories indirectly (e.g., through error detection and/or correction) is of major interest to the field of motor control.

Recently, work in rodents has shown that neural plasticity induced by maze training is reflected in macroscopical changes in

\footnotetext{
Received May 5, 2011; revised June 10, 2011; accepted June 16, 2011.

Author contributions: V.D.-M. designed research; S.M.L. performed research; F.B. contributed unpublished reagents/analytic tools; S.M.L. analyzed data; S.M.L. and V.D.-M. wrote the paper.

The study was supported by the Fogarty International Research Collaboration Award (NIH) and a grant from the National Agency for the Promotion of Science and Technology (ANPCyT). We thank Scott Grafton, Kate Watkins, and Gustavo Murer for their suggestions and valuable feedback. We also thank Dr. Martin Eleta for allowing us access to the MRI facility in TCBA.

Correspondence should be addressed to Valeria Della-Maggiore, Department of Physiology and Biophysics, School of Medicine, University of Buenos Aires, C1121ABG Buenos Aires, Argentina. E-mail: vdellamaggiore@fmed.uba.ar.

DOI:10.1523/JNEUROSCI.2253-11.2011

Copyright $\odot 2011$ the authors $\quad 0270-6474 / 11 / 3111808-06 \$ 15.00 / 0$
}

hippocampal structure that can be detected with magnetic resonance imaging (MRI) (Lerch et al., 2011). MRI provides, among other measures, information regarding gray matter concentration (GMC) and white matter microstructure (e.g., fractional anisotropy, FA) (e.g., Scholz et al., 2009). The present study was designed to identify potential sites of neural plasticity induced by adaptation. For this purpose, we used MRI to detect structural changes in GMC and FA after 1 week of intensive training on a visuomotor adaptation task involving a complex optical rotation. We have previously shown that this task is associated with savings at test 1 month later, and with learning-related increments in the functional connectivity among the anterior cerebellum, contralateral M1, and PPC (Della-Maggiore and McIntosh, 2005).

In light of our functional study and the literature reviewed above, we predict that the same training protocol will induce structural plasticity in these regions and associated fiber tracts. Nevertheless, due to their differential time of recruitment, we expect structural changes within M1 and PPC to correlate with the rate of adaptation during late stages of learning and cerebellar changes to correlate with the rate of adaptation during early stages of learning. Recent evidence suggests that short-term motor training may lead to long-term retention. For example, new visuomotor maps learned over a few trials can be retained beyond several months (Yamamoto et al., 2006). This is consistent with the demonstration that new dendritic spines generated in M1 during early motor training persist up to 3 months (Yang et al., 2009). Based on these reports and the functional evidence discussed above supporting a role of M1 in motor memory consolidation, we hypothesize that cortical increments in M1 would predict savings 1 year later. 


\section{Materials and Methods \\ Participants}

Twelve healthy right-handed volunteers (age range 22-35, mean \pm SD: $25.5 \pm 4.1$ years, 6 females) participated in the study. They did not present any neurological or psychiatric disorders. The experimental procedure was approved by the Ethics Committee of the University of Buenos Aires Hospital and performed according to the Declaration of Helsinki.

\section{Experimental paradigm}

This work was designed as a follow up of our functional study, where we examined changes in functional connectivity induced by adaptation (Della-Maggiore and McIntosh, 2005). Here, we used the same experimental paradigm and a very similar training protocol $(7 \mathrm{~d}$ of training instead of 6) to explore the possibility that increased functional connectivity was accompanied by structural plasticity. For a full description of the task and the complexity of the perturbation refer to our previous study. Briefly, participants tracked a target moving semirandomly on a computer screen for 9 consecutive days using a MRI-compatible joystick controlled with the thumb and index fingers of their right hand. The hand was represented on the screen by a cursor. Subjects were instructed to keep the distance between target and cursor minimal at all times. During the first day of the experiment, subjects performed a familiarization session (42 $\mathrm{min}$ ) to become familiar with the visuomotor tracking task and the joystick. We have previously shown that this amount of practice is sufficient to achieve asymptotic performance (DellaMaggiore, 2003; Della-Maggiore and McIntosh, 2005). Familiarization was, thus, crucial to ensure that longitudinal changes in brain structure were likely due to visuomotor adaptation and not to nonspecific effects associated with learning the tool and/or the task. The last block of this session was used as a baseline to adjust for interindividual differences in the level of performance (see below, Data analysis). Training on the visuomotor adaptation task took place during the following $7 \mathrm{~d}$. On the first day of training a complex perturbation that distorted visuomotor coordination was applied. This consisted of an optical rotation of the cursor, which varied depending on movement direction along the horizontal axis of the joystick. Consequently, the direction of movement on the screen was the mirror image of the hand real movement direction. This complex perturbation increased task difficulty prolonging the time to adaptation beyond one session typically observed in standard centerout-back tasks (Krakauer, 2009). Given that the target moved fast and smoothly, participants were unable to figure out the optical rotation as assessed by debriefing. The day immediately after the end of training, veridical visual feedback was restored. This procedure, also known as washout, eliminates the behavioral manifestation of learning thereby allowing the measurement of savings. Savings, defined as the impact of previous learning on faster readaptation upon a second encounter with the perturbation, were assessed at test $\sim 1$ year later. Daily practice during the familiarization, training, washout, and test sessions consisted of six 7 min blocks separated by 2 min rest blocks ( $42 \mathrm{~min}$ of training per day).

\section{MRI data acquisition}

All images were acquired on a 1.5 tesla Philips Intera scanner.

\section{Structural images}

High-resolution $\mathrm{T} 1$ images $(\mathrm{TR}=25 \mathrm{~ms} ; \mathrm{TE}=4.954 \mathrm{~ms}$; matrix $=$ $256 \times 256 ;$ FOV $=256$; flip angle $=30$; voxel size $=1 \times 1 \times 1 \mathrm{~mm}^{3} ; 160$ slices) and diffusion-weighted images (DWI, 3 volumes without diffusion +3 acquisitions of 32 directions; $b=800 \mathrm{~s} \cdot \mathrm{mm}^{-2}$; matrix $=112 \times$ $112 ; \mathrm{FOV}=224 ; 70$ slices; voxel size $2 \times 2 \times 2 \mathrm{~mm}$; $\mathrm{TR}=9.9 \mathrm{~s}$; $\mathrm{TE}=$ $80 \mathrm{~ms}$ ) were obtained before introducing the perturbation on the first day training (scan 1) and after adaptation on the seventh day of training (scan 2).

\section{Functional images}

In addition, 203 functional echo-planar images (EPI; TR $=3.2 \mathrm{~s} ; \mathrm{TE}=50$ ms; matrix $=64 \times 64 ; \mathrm{FOV}=256$, voxel size $=4 \times 4 \times 4 \mathrm{~mm}, 36$ slices $)$ were acquired before adaptation on day 1 as subjects performed the task without the perturbation, following a block design of $20 \mathrm{~s} \mathrm{ON,} 12.5 \mathrm{~s}$ OFF

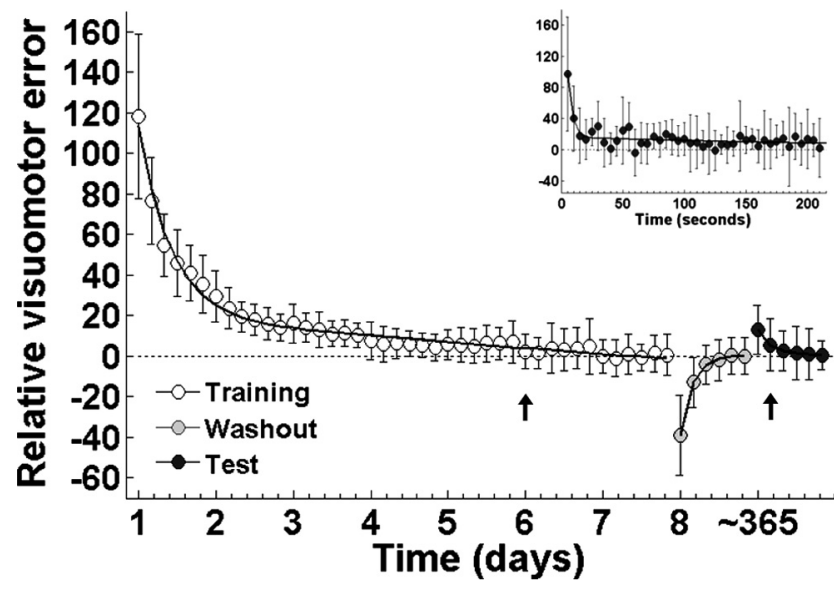

Figure 1. Time course of visuomotor error as a function of time. Symbols represent mean \pm SD for visuomotor error. Error was fit with a double exponential function for adaptation (left, white symbols), washout (middle, gray symbols), and test (right, black symbols). Baseline is depicted by a dotted straight line. Time to adaptation both during adaptation and test is depicted by an arrow. Inset shows a detail of the decay in visuomotor error (averaged every $5 \mathrm{~s}$ ) during the first block of readaptation at test.

(total number of blocks $=20$ ). Participants viewed the task through a mirror. Subjects performed the task during ON blocks and fixated on a central cross during OFF blocks. Due to an artifact, data from 10 of 12 subjects was analyzed.

\section{Data analysis: Behavior}

The distance between target and cursor (sampled at $200 \mathrm{~Hz}$ ), was used as an index of visuomotor error, which was averaged for each block and expressed as a percentage of the baseline for each subject. This latter step was performed to account for interindividual differences in the level of performance. The average baseline is shown in Figure 1 as the straight dotted line. To contrast the time course of adaptation, washout, and readaptation at test in the same figure, the baseline was set to zero. Oneway repeated-measures ANOVA (RM-ANOVA) with time as factor was performed on the visuomotor error during training and test $(n=12)$. Time to adaptation and readaptation at the group level was defined as the first block in which error did not differ significantly from the baseline, and was determined by Tukey's HSD test ( $\alpha$ was set at 0.1 ).

\section{Behavioral measures of interest}

Fast and slow rate of adaptation. To characterize the rate of adaptation, each individual's visuomotor error (the mean for each 7 min block) was fit with a double exponential function, characteristic of this type of learning $\left[f(t)=k_{1} \times e^{\lambda 1 \times t}+k_{2} \times e^{\lambda 2 \times t}\right.$, where $t$ represents 7 min bins (Smith et al., 2006)]. Given that the three potential regions for plasticity appear to be recruited differentially during adaptation, we used the fast and slow coefficients of this function to capture the rate of learning during early and late stages of adaptation, respectively. These coefficients were in turn correlated with longitudinal changes in GMC and FA to identify learning-specific plasticity.

Savings. The amount of savings was estimated from the mean error exhibited during the first 7 min block of readaptation at test around 1 year later, the rationale being that a small error would reflect faster readaptation than a large error. This was an indirect way of estimating the speed of readaptation during the first block, which could not be assessed in some subjects by fitting an exponential function because they adapted immediately. Longitudinal changes in GMC and FA were correlated with this variable to examine whether structural changes impacted on the level of performance on a large time scale.

For each MRI measure, brain-behavior correlations were adjusted for multiple comparisons using Bonferroni's correction ( $\alpha$ was set at 0.017 ).

\section{Potential confounds}

Time of test. Subjects underwent the test session at different times about a year after the training $(480.54 \pm 27.63 \mathrm{~d})$, introducing forgetting as a 
potential confound when measuring correlations with savings. To rule out this possibility, we computed the correlation between the increment in structural measures and time of test.

Initial visuomotor skill. To discard that correlations between structural measures and savings were driven by individual differences in the initial level of skill with the joystick, we computed the relationship between baseline (familiarization session) and structural changes.

\section{Image preprocessing and statistical analysis Diffusion-weighted images}

The analysis of DWI images was performed in the whole brain using FSL v.4.1 (fMRIB Software Library). DWI images were first eddy current and motion corrected and skull stripped. Then, FA images were created by fitting a tensor model to the DWI images using $F D T$, and normalized to the midspace between the two scans (Scholz et al., 2009). Longitudinal increases in FA induced by training were examined by contrasting scan 2 - scan 1 after covarying for age, which has significant effects on FA (Pfefferbaum et al., 2000). A height threshold was set at $t>4.02$, corresponding to an uncorrected $p$ value of 0.001 , followed by a cluster threshold of $p<0.05$, corrected for multiple comparisons. The difference in FA obtained from subtracting scan 1 from scan 2 for the peak voxel of each cluster was used to compute correlations with the behavioral measures.

To identify white matter tracts associated with changes in FA, probabilistic tractography was performed using the voxels of the clusters identified with TBSS on FA images as seed masks (Behrens et al., 2007). This method has been reported in full previously (Behrens et al., 2003, 2007). Briefly, a multifiber diffusion model was used to estimate the probability distributions of the direction of fiber populations passing through each brain voxel. Tractography then proceeded by drawing 25,000 streamline samples from each seed voxel to build up an estimate of the connectivity distribution. Waypoints were used when necessary to constrain the probabilistic tractography to those fibers passing through them (see Results). The number of the samples passing through each brain voxel can be interpreted as the probability of connection to the seed voxel. Pathways in individual subjects were thresholded to include only voxels that had at least 25 samples allowing sensitivity to weak paths while leaving out spurious connections. Paths identified in this way were binarized for each subject and then overlaid to produce population probability maps for each pathway reflecting the proportion of the population in which a tract was present.

\section{T1-weighted images}

The analysis of T1 images was performed with SPM5 (Wellcome Department of Cognitive Neurology, UCL). Images were registered to the midway space between scan 1 and 2 using an affine transformation. Then a voxel-based morphometry (VBM) analysis was performed to detect changes in GMC following a unified tissue segmentation and nonlinear registration procedure. To correct for local geometric expansions or contractions arising during spatial normalization, images were modulated using the deformation field of the transformation. Finally, given that the thickness of the human cerebral cortex varies between 1 and $4.5 \mathrm{~mm}$ (Fischl and Dale, 2000), the resulting gray matter images were smoothed with an isotropic Gaussian kernel of $4 \mathrm{~mm}$ FWHM.

SnPM5 was used for statistical assessment on a search volume defined by the functional mask obtained from contrasting ON versus OFF fMRI blocks using a voxelwise, random-effect analysis (SPM5). This mask included the contralateral sensorimotor, posterior parietal, dorsal premotor cortex, and bilateral cerebellum (Fig. 2). To ensure a good brain coverage, we set an uncorrected height threshold to $p<0.001$. For the statistical analysis of T1 images, increments in GMC induced by training were assessed by contrasting scan $2-$ scan 1 . A height threshold was set at $t>4.02$ (corresponding to an uncorrected $p$ value of 0.001 ) followed by a cluster threshold of $p<0.05$, corrected for multiple comparisons. The difference in GMC obtained from subtracting scan 1 from scan 2 for the peak voxel of each cluster was used to compute correlations with the behavioral measures.

\section{Results}

\section{Behavioral results}

On average, adaptation was achieved during the first block of the sixth day of training (Fig. 1, leftmost plot) (one-way RMANOVA, $F_{(42,462)}=75.699, p<0.0001$, followed by Tukey's HSD $p=0.402)$. After training, the optical rotation was removed (washout) revealing aftereffects consistent with the formation of a motor memory (Shadmehr and Mussa-Ivaldi, 1994) (Fig. 1, middle plot, gray symbols).

In contrast to the $6 \mathrm{~d}$ needed to reach adaptation, readaptation assessed at test was achieved on the second block, indicating the presence of savings despite the long interval without practice (Fig. 1, rightmost plot and inset, black symbols) (one-way RMANOVA, $F_{(6,66)}=16.024, p<0.0001$, followed by Tukey's HSD $p=0.354)$. To further determine the exact amount of time required to readapt, we ran a RM-ANOVA with minutes instead of blocks as the time factor. Subjects took $8 \mathrm{~min}$ to readapt. In other words readaptation was achieved in the first minute of the second test block $\left(F_{(42,462)}=3.37, p=0.008\right.$, followed by Tukey's HSD $p=0.104)$.

\section{MRI results}

Gray matter concentration and FA are increased by visuomotor adaptation

Training induced a significant increment in GMC only in a region of the left primary motor cortex corresponding to the hand representation (peak voxel: $t=4.73$, MNI coordinates $-34-18$ 46 , spatial extent $=16$ voxels, $p<0.05$, corrected for multiple comparisons) (Fig. 3, top). Three pieces of evidence suggest that changes in GMC across scans were learning specific and not due to a systematic bias in $\mathrm{T} 1$ weighting toward one scanning session. First, voxel intensity was normalized relative to the corresponding volume. Second, T1 images for each subject were acquired on different days, reducing the possibility of a systematic bias. Finally, changes in GMC were effector specific (hand area of M1), and were not observed in other regions of the cortex.

In addition, training induced a significant increment in FA only in one region located below the GMC cluster identified in the VBM analysis (peak $t=6.07$, MNI coordinates $-34-2137$, spatial extent $=22$ voxels, $p<0.05$, corrected for multiple comparisons) (Fig. 3 , middle).

There was no significant correlation between gray and white matter changes across subjects $(r=0.27, p=0.387)$. 


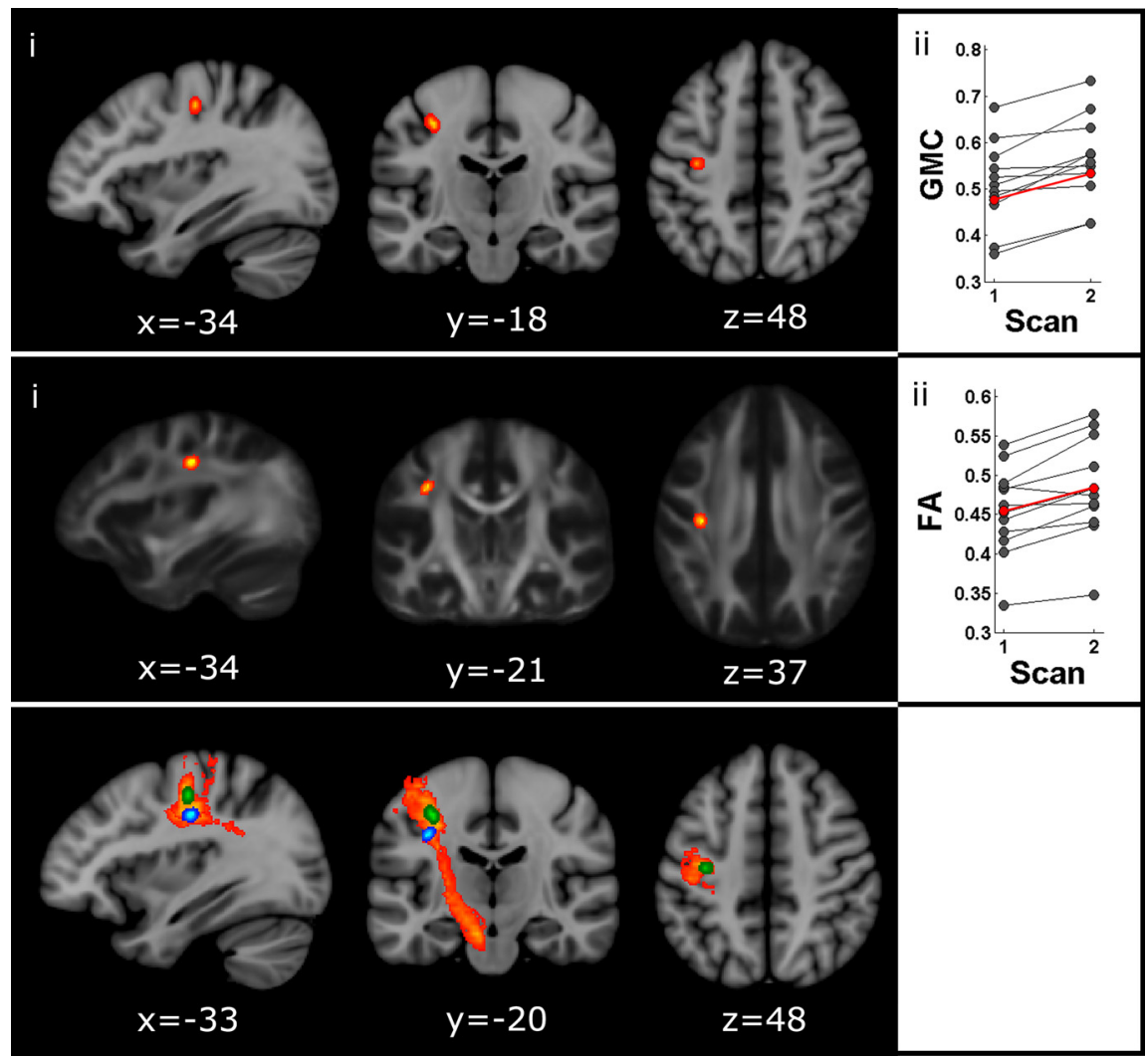

Figure 3. Structural changes induced by motor adaptation. Top, Effect of adaptation on gray matter concentration. $\boldsymbol{i}$, Region of the left M1 (hand area) showing an increase in GMC with learning. Results are overlaid on the MNI 152 T1 template. ii, Individual (black) and mean changes (red) in GMC across scanning sessions. Middle, Effects of adaptation on fractional anisotropy. $\boldsymbol{i}$, White matter region showing an increase in FA with learning. Results are overlaid on the FA template (FSL). $\boldsymbol{i}$, Individual (black) and mean changes (red) in FA across scanning sessions. Bottom, Probabilistic tractography. White matter fibers connecting the FA cluster (blue) with the GMC cluster (green) and the cerebral peduncle (likely, corticospinal fibers). Paths are group probability maps, where color coding reflects the proportion of the population in which a tract is present from red (3/12) to yellow (12/12). Results are overlaid on the MNI $152 \mathrm{~T} 1$ template. Image orientation is radiological.
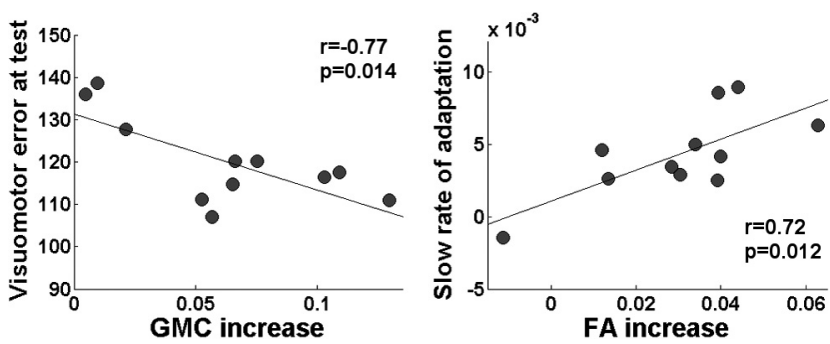

Figure 4. Relationship between structural changes, learning and savings. Left, Correlation between increments in GMC induced by training (cluster peak voxel) and savings measured 1 year later. Right, Correlation between increments in FA induced by training (cluster peak voxel) and the slow rate of adaptation.

\section{Probabilistic tractography}

Given that (1) the location of the FA cluster was anatomically compatible with the route of descending pathways, (2) the corticospinal tract originates partly in M1, and (3) GMC cluster was localized to the hand representation of M1, we ran a tractography to explore whether both clusters laid on the same tract. The FA cluster was used as a seed and the cerebral peduncle as a waypoint to identify major descending pathways such as the corticospinal tract. Colocalization of FA and GMC clusters to the same descending paths (Fig. 3, bottom) suggests a functional link between the mechanisms driving structural plasticity. We cannot discard, however, that FA changes affected other tracts following a similar route that were not tested here such as the corticothalamic or corticostriatal.

\section{Relationship between structural} changes, rate of learning, and savings Correlation between longitudinal changes in GMC, savings, and the rate of adaptation

Increments in GMC were significantly correlated with savings at test $(r=-0.77$, $p=0.014$ ) (Fig. 4). In other words, participants who showed greater GMC after training adapted more rapidly during a second encounter with the same perturbation 1 year later. On the contrary, no significant correlation was found between the increment in GMC and the speed of learning across individuals $(r=-0.23$, $p=0.49$ for the fast and $r=-0.46, p=$ 0.16 for the slow coefficient).

In addition, no significant correlation was found between increments in GMC with time of test $(r=0.26, p=0.43)$ or with the initial visuomotor skill $(r=0.24$, $p=0.47)$, ruling out these factors as possible confounds.

\section{Correlation between longitudinal changes} in FA, savings, and the rate of adaptation Increments in FA were positively correlated with the slow coefficient of the double exponential during adaptation $(r=$ $0.72, p=0.012$ ) (Fig. 4). In other words, faster adaptation during late stages of learning was associated with larger increments in FA. No significant correlations were found between changes in FA and the fast rate of learning $(r=0.39, p=0.23)$ or savings $(r=-0.0176, p=0.96)$.

\section{Discussion}

In this study, we used MRI to identify macroscopical correlates of structural plasticity induced by adaptation. One week of motor training led to an increment in gray matter concentration over the hand area of the contralateral primary motor cortex and an increase in fractional anisotropy of white matter fibers underneath this region. Tractography suggested that structural changes colocalized to descending tracts (e.g., corticospinal pathway), suggesting a functional link between the mechanisms driving these events. We have previously shown that 1 week of training on the same paradigm induces learning-related changes in the functional connectivity of a network including M1, PPC, and cerebellum (Della-Maggiore and McIntosh, 2005). The demonstration that the same experimental protocol induces structural changes in M1 strengthens our previous work and provides further evidence supporting a role of this region in motor plasticity.

Congruent evidence from human and nonhuman animals points to the primary motor cortex as one of the key regions in motor adaptation. Electrophysiological studies in monkeys have shown that reaching adaptation to proprioceptive and visual perturbations (Li et al., 2001; Paz et al., 2003) shifts the preferred direction of neurons in M1 to encode the direction opposite to the perturbation. The persistence of these changes through wash- 
out and at test is compatible with the formation of motor memories. This hypothesis finds support in human studies showing that disruption of M1 function by transcranial magnetic stimulation interferes with readaptation $24 \mathrm{~h}$ later (Richardson et al., 2006).

Our findings are consistent with the literature on motor adaptation reviewed above and shed new light on the neural substrates of motor learning. On the one hand, we demonstrate for the first time that adaptation is associated with structural changes in M1. The colocalization of gray and white matter clusters to the same pathways reinforces the anatomical specificity of our findings. On the other hand, the fact that increments in GMC predicted the level of savings 1 year later strongly suggests that M1 may be part of a network where motor memories for the new visuomotor map are stored. To the best of our knowledge, this is the first demonstration that early structural changes induced by motor learning may impact on behavior after such a long period without training. Further work would be needed to establish a causal relationship between increments in GMC and savings.

Recently, Smith et al. (2006) proposed a two-module learning model to explain motor adaptation: the fast module is very sensitive to error but forgets rapidly, whereas the slow module is not as sensitive to error but has very good retention. The facts that changes in white matter microstructure correlated with the slow learning coefficient and that structural changes in M1 correlated with savings 1 year later support a role of this region as part of the slow learning module.

In addition to M1, we predicted structural changes in the cerebellum and the PPC, which showed sustained increments in functional connectivity in our previous study (Della-Maggiore and McIntosh, 2005). One explanation for the mismatch between our findings and our predictions may lie in a prevalent role of M1 in motor memory consolidation and/or storage. In contrast, the cerebellum and the PPC may contribute to the formation of new motor memories indirectly through error correction and/or detection (e.g., state estimation) (Desmurget et al., 1999; Miall et al., 2007) without undergoing plasticity. Alternatively, the limiting resolution of human T1 images $(1 \times 1 \times 1 \mathrm{~mm})$ may have compromised the detection of changes particularly in the cerebellum, of which the cortex is $\sim 1 \mathrm{~mm}$.

Longitudinal increments in GMC and FA have previously been reported for other types of motor learning such as juggling (Draganski et al., 2004; Driemeyer et al., 2008; Scholz et al., 2009). However, neither performance nor exercise alone could explain the extent of these structural changes. Longitudinal alterations in the amount of gray and white matter have also been detected in supplementary motor area and left prefrontal regions during 6 weeks of training on a whole-body balancing task. Yet, only prefrontal changes were correlated with improvements in performance during the learning period (Taubert et al., 2010). Our work provides the first evidence indicating that motor training in adult humans induces structural changes that predict faster relearning way beyond the period of practice. A previous study using a similar adaptation task spread in six 25 min training sessions over a two-week schedule failed to find structural changes in GMC (Thomas et al., 2009). We believe that the intensity and/or repetition of training chosen here were, thus, crucial to the induction of more dramatic changes in gray matter. This is the case in rats, where synaptogenesis in M1 is elicited after 400 but not 60 trials of motor skill training per day (Kleim and Jones, 2008). In addition to the intensity and repetition of training, the timing of image acquisition chosen here, 1 week after training onset, may have also contributed to the detection of learning-related structural changes. This is in line with recent evidence suggesting that a few days of training are enough to induce new synaptic spines that relate to gains in performance within a time scale of days (Yang et al., 2009). This critical period may explain why juggling studies, in which subjects were scanned after longer periods of practice, failed at detecting learningrelated structural changes.

Little is known about the correspondence between structural plasticity induced by learning and macroscopic alterations detected with MRI. Work from rodents indicates that skilled reaching and rotarod learning induce new dendritic spines in M1 (Xu et al., 2009; Yang et al., 2009) and may be accompanied by glial changes such as astrocyte hypertrophy (Kleim et al., 2007). One possible explanation for the absence of a relationship between longitudinal increments in gray matter concentration of M1 and the slow rate of learning could be that the occurrence of these biological events is influenced, not necessarily by the rate of learning, but by other factors such as the intensity and/or total amount of practice (Kleim and Jones, 2008). Further experiments would be needed to assess this possibility. As for the biological basis of macroscopical alterations in white matter, several factors may have led to longitudinal changes in FA. Myelination, axonal packing density, and axon diameter may influence fractional anisotropy (Beaulieu, 2002). The colocalization of WM and GM clusters to the same projection fibers opens the possibility that functional changes in the efficacy of motor circuits-such as LTP — may have led to axonal remodeling and/or myelination (Demerens et al., 1996). The fact that the identified FA cluster was localized to descending pathways and correlated positively with the slow rate of learning but not with savings points to myelination as a possible biological substrate of the reported changes in white matter microstructure.

Despite its strengths, our study had two main limitations. On the one hand, no scan was acquired at test, which would have strengthened further our findings. Nevertheless, evidence shows that longitudinal changes in GMC induced by juggling recede when assessed after a period of 2-4 months without training (Draganski et al., 2004; Driemeyer et al., 2008), suggesting that human MRI may not be sensitive enough to detect persistent plastic changes 1 year after the end of practice. On the other hand, there was no control group. Yet, the fact that subjects had previous experience with the visuomotor tracking task during the familiarization session, which is sufficient to reach asymptotic performance (Della-Maggiore, 2003; Della-Maggiore and McIntosh, 2005), suggests that nonspecific learning took place before the first MRI session. This point is further strengthened by our previous demonstration that $6 \mathrm{~d}$ of training without the optical rotation induced no changes in performance or functional connectivity within the motor network (Della-Maggiore and McIntosh, 2005). Although the occurrence of nonspecific effects cannot be ruled out, the evidence presented above together with the strong relationship found between structural changes, savings, and the speed of learning suggests that our findings indeed related to adaptation.

In conclusion, we have shown that 1 week of intensive visuomotor adaptation may impact the cortical motor circuitry supporting long-term memory for 1 year. Moreover, we present evidence suggesting that this type of learning is accompanied by structural changes in descending pathways connecting cortical circuits with lower-level motor centers, such as the corticospinal tract. Our results are in line with current electrophysiological studies in monkeys pointing to the contralateral primary motor cortex as a main site of plasticity during motor adaptation. We 
propose that the human brain adapts to a continuously changing environment by modifying the structure of specific motor circuits with a long-lasting impact on behavior. This hypothesis is now amenable to testing.

\section{References}

Beaulieu C (2002) The basis of anisotropic water diffusion in the nervous system-a technical review. NMR Biomed 15:435-455.

Behrens TE, Woolrich MW, Jenkinson M, Johansen-Berg H, Nunes RG, Clare S, Matthews PM, Brady JM, Smith SM (2003) Characterization and propagation of uncertainty in diffusion-weighted MR imaging. Magn Reson Med 50:1077-1088.

Behrens TE, Berg HJ, Jbabdi S, Rushworth MF, Woolrich MW (2007) Probabilistic diffusion tractography with multiple fibre orientations: what can we gain? Neuroimage 34:144-155.

Criscimagna-Hemminger SE, Bastian AJ, Shadmehr R (2010) Size of error affects cerebellar contributions to motor learning. J Neurophysiol 103:2275-2284.

Della-Maggiore V (2003) Large-scale plasticity: learning-related changes in brain activity and functional connectivity associated with adaptation to distortion of visual feedback. $\mathrm{PhD}$ thesis, University of Toronto.

Della-Maggiore V, McIntosh AR (2005) Time course of changes in brain activity and functional connectivity associated with long-term adaptation to a rotational transformation. J Neurophysiol 93:2254-2262.

Della-Maggiore V, Malfait N, Ostry DJ, Paus T (2004) Stimulation of the posterior parietal cortex interferes with arm trajectory adjustments during the learning of new dynamics. J Neurosci 24:9971-9976.

Demerens C, Stankoff B, Logak M, Anglade P, Allinquant B, Couraud F, Zalc $B$, Lubetzki C (1996) Induction of myelination in the central nervous system by electrical activity. Proc Natl Acad Sci U S A 93:9887-9892.

Desmurget M, Epstein CM, Turner RS, Prablanc C, Alexander GE, Grafton ST (1999) Role of the posterior parietal cortex in updating reaching movements to a visual target. Nat Neurosci 2:563-567.

Draganski B, Gaser C, Busch V, Schuierer G, Bogdahn U, May A (2004) Neuroplasticity: changes in grey matter induced by training. Nature 427:311-312.

Driemeyer J, Boyke J, Gaser C, Büchel C, May A (2008) Changes in gray matter induced by learning-revisited. PLoS ONE 3:e2669.

Fischl B, Dale AM (2000) Measuring the thickness of the human cerebral cortex from magnetic resonance images. Proc Natl Acad Sci U S A 97:11050-11055.

Kleim JA, Jones TA (2008) Principles of experience-dependent neural plasticity: implications for rehabilitation after brain damage. J Speech Lang Hear Res 51:S225-239.

Kleim JA, Markham JA, Vij K, Freese JL, Ballard DH, Greenough WT (2007) Motor learning induces astrocytic hypertrophy in the cerebellar cortex. Behav Brain Res 178:244-249.

Krakauer JW (2009) Motor learning and consolidation: the case of visuomotor rotation. Adv Exp Med Biol 629:405-421.

Lerch JP, Yiu AP, Martinez-Canabal A, Pekar T, Bohbot VD, Frankland PW,
Henkelman RM, Josselyn SA, Sled JG (2011) Maze training in mice induces MRI-detectable brain shape changes specific to the type of learning. Neuroimage 54:2086-2095.

Li CS, Padoa-Schioppa C, Bizzi E (2001) Neuronal correlates of motor performance and motor learning in the primary motor cortex of monkeys adapting to an external force field. Neuron 30:593-607.

Martin TA, Keating JG, Goodkin HP, Bastian AJ, Thach WT (1996) Throwing while looking through prisms. I. Focal olivocerebellar lesions impair adaptation. Brain 119:1183-1198.

Miall RC, Christensen LO, Cain O, Stanley J (2007) Disruption of state estimation in the human lateral cerebellum. PLoS Biol 5:e316.

Orban de Xivry JJ, Criscimagna-Hemminger SE, Shadmehr R (2011) Contributions of the motor cortex to adaptive control of reaching depend on the perturbation schedule. Cereb Cortex 21:1475-1484.

Paz R, Boraud T, Natan C, Bergman H, Vaadia E (2003) Preparatory activity in motor cortex reflects learning of local visuomotor skills. Nat Neurosci 6:882-890.

Pfefferbaum A, Sullivan EV, Hedehus M, Lim KO, Adalsteinsson E, Moseley M (2000) Age-related decline in brain white matter anisotropy measured with spatially corrected echo-planar diffusion tensor imaging. Magn Reson Med 44:259-268.

Richardson AG, Overduin SA, Valero-Cabré A, Padoa-Schioppa C, PascualLeone A, Bizzi E, Press DZ (2006) Disruption of primary motor cortex before learning impairs memory of movement dynamics. J Neurosci 26:12466-12470.

Scholz J, Klein MC, Behrens TE, Johansen-Berg H (2009) Training induces changes in white-matter architecture. Nat Neurosci 12:1370-1371.

Shadmehr R, Mussa-Ivaldi FA (1994) Adaptive representation of dynamics during learning of a motor task. J Neurosci 14:3208-3224.

Smith MA, Shadmehr R (2005) Intact ability to learn internal models of arm dynamics in Huntington's disease but not cerebellar degeneration. J Neurophysiol 93:2809-2821.

Smith MA, Ghazizadeh A, Shadmehr R (2006) Interacting adaptive processes with different timescales underlie short-term motor learning. PLoS Biol 4:e179.

Taubert M, Draganski B, Anwander A, Müller K, Horstmann A, Villringer A, Ragert P (2010) Dynamic properties of human brain structure: learning-related changes in cortical areas and associated fiber connections. J Neurosci 30:11670-11677.

Thomas AG, Marrett S, Saad ZS, Ruff DA, Martin A, Bandettini PA (2009) Functional but not structural changes associated with learning: an exploration of longitudinal voxel-based morphometry (VBM). Neuroimage $48: 117-125$

Xu T, Yu X, Perlik AJ, Tobin WF, Zweig JA, Tennant K, Jones T, Zuo Y (2009) Rapid formation and selective stabilization of synapses for enduring motor memories. Nature 462:915-919.

Yamamoto K, Hoffman DS, Strick PL (2006) Rapid and long-lasting plasticity of input-output mapping. J Neurophysiol 96:2797-2801.

Yang G, Pan F, Gan WB (2009) Stably maintained dendritic spines are associated with lifelong memories. Nature 462:920-924. 